\title{
SUR LES ALGEBRES DE BERNSTEIN V: LES ALGEBRES DE BERNSTEIN JORDAN FAIBLES*
}

\author{
by ALICIA LABRA, CRISTIAN MALLOL, ARTIBANO MICALI \\ et RICHARD VARRO
}

(Received 16th April 1990)

\begin{abstract}
Nous présentons ici l'étude, dans l'optique exhibée dans [7], d'une catégorie d'algèbres à idempotents, commutatives, non associatives, définies par des relations. On y trouve une structure qui n'est en général ni pondérée ni de Jordan et oú, cependant, ces conditions sont équivalentes.

Following a previous paper, we study here a class of commutative and non associative algebras with idempotents and defined by relations. We find a structure which is not, in general, baric or Jordan but where these notions are equivalent.
\end{abstract}

1980 Mathematics subject classification (1985 Revision). 17D92.

\section{Introduction}

Dans le contexte de la modélisation des situations issues de la génétique, la notion de structure d'algèbre s'est montrée efficace. Ainsi sont apparues quelques algèbres pondérées, i.e. la donnée d'un couple $(A, \omega)$ où $A$ est une $K$-algèbre et $\omega: A \rightarrow K$ un morphisme non nul d'algèbre.

A partir des travaux de Serge Bernstein en 1923 (c.f. $[1,2,3]$ ), où il démontre que la seule loi de l'hérédité compatible avec le principe de stationnarité est la loi de Mendel, la notion d'algèbre de Bernstein a été introduite [6]; elle a été développée [4] puis généralisée [8]: une algèbre pondérée $(A, \omega)$ est de Bernstein d'ordre $n$, si pour tout élément $y$ dans $A$ on a: $y^{[n+2]}=[\omega(y) y]^{[n+1]}$, l'entier $n$ étant le plus petit entier pour lequel cette identité est vérifiée.

Enfin, introduites dans la formalisation de la mécanique quantique, les algèbres de Jordan sont définies par l'identité $(x y) x^{2}=x\left(y x^{2}\right)$. Les algèbres de Bernstein-Jordan ont été étudiées dans [9] et sont caractérisées de façon biunivoque par l'identité $y^{3}=\omega(y) y^{2}$.

\section{Définition et équivalences}

Soient $K$ un corps commutatif et $A$ une $K$-algèbre commutative de dimension finie.

* Subventioné par FONDECYT (proyecto 0227/90) et par le Departamento Técnico de Investigacion, Universidad de Chile (proyecto E-2585/9044). 
On dit que $A$ est Bernstein Jordan Faible (BJF) si elle admet une décomposition $A=\mathrm{Ke} \oplus S$ telle que e est un idempotent non nul et pour tout $x \in S$ on a les relations:

$$
\begin{gathered}
e x=2 e(e x), \\
x^{2}=e x^{2}+2(e x) x \quad \text { et } \\
x^{3}=0 .
\end{gathered}
$$

Si l'on considère l'algèbre $A$ étant associative, on a $e S=S^{2}=\{0\}$; elle est donc constante, pondérée et vérifie l'identité $y^{2}=\omega(y)^{2} e$ pour tout $y \in A$ où $\omega$ est le morphisme d'algèbres de $A$ dans $K$ défini par $\omega(\lambda e+x)=\lambda$ avec $x \in S$.

De même, si la caractéristique du corps est 2 on a $e S=\{0\}$ et $x^{2} \in \mathrm{Ke}$ pour tout $x \in S$; ainsi $A$ est constante et admet une pondération si et seulement si $S^{2}=\{0\}$.

Dorénavant, le corps $K$ est infini de caractéristique différente de 2 et l'algèbre $A$ sans condition d'associativité. De ce fait, par les techniques de linéarisation et polarisation, des relations 2.2 et 2.3 ont obtient respectivement les relations:

$$
\begin{gathered}
x y=e(x y)+(e x) y+(e y) x \\
(x y) z+(z x) y+(z y) x=0, \text { pour tout } x, y \text { et } z \text { dans } S .
\end{gathered}
$$

Exemple 2.6. Considérons l'algèbre $A$ de base $\left\{x_{1}, \ldots, x_{5}\right\}$ et de table de multiplication:

$$
x_{1}^{2}=x_{1}, x_{1} x_{2}=\frac{1}{2} x_{2}, x_{1} x_{3}=\frac{1}{2} x_{3}, x_{2}^{2}=x_{4}, x_{3}^{2}=\frac{\lambda}{4} x_{4},
$$

$x_{5}^{2}=\lambda x_{1}, x_{2} x_{5}=x_{3}, x_{3} x_{5}=-\frac{\lambda}{4} x_{2}$ avec $\lambda \in K, \lambda \neq 0$, les autres produits étant nuls.

Cette algèbre est $\mathrm{BJF}$, non associative, non pondèrable ni de Jordan.

Exemple 2.7. Le Théorème 2.8 montre que toute algèbre pondérée $(A, \omega)$ de Bernstein-Jordan (d'ordre $\leqq 1$ ) est une algèbre BJF, justifiant ainsi le nom choisi pour ce type d'algèbre.

Théorème 2.8. Soit $A$ une algèbre commutative. Alors $A$ est $B J F$ si et seulement si il existe une forme linéaire $\omega$ et un élément $\varepsilon$ de $A$ tels que $\omega\left(\varepsilon^{3}\right) \neq 0$ et $y^{3}=\omega(y) y^{2}$ pour tout $y$ dans $A$.

Démonstration. Si $A$ est une algèbre BJF, on a $e^{3}=e \neq 0$; de plus, si $y=\lambda e+x$ est un élément de $A$, un calcul simple, à l'aide des relations 2.1, 2.2 et 2.3, permet d'établir que $y^{3}=\lambda y^{2}$. Il suffit, donc, de poser $\omega(y)=\lambda$. Réciproquement, soit $A$ une algèbre vérifiant les conditions de l'énoncé ci-dessus. Montrons que $A$ admet un idempotent non nul: en effet, de l'identité $(x+y)^{3}=\omega(x+y)(x+y)^{2}$ on obtient $2 x(x y)+x^{2} y=2 \omega(x) x y+$ $\omega(y) x^{2}\left(^{*}\right)$ (par polarisation). Puis, soit $\varepsilon$ un élément de $A$ tel que $\omega\left(\varepsilon^{3}\right) \neq 0$; or, 
$\varepsilon^{3}=\omega(\varepsilon) \varepsilon^{2}$ alors $\omega(\varepsilon) \neq 0, \omega\left(\varepsilon^{2}\right) \neq 0$ et on peut supposer $\omega(\varepsilon)=1$ (il suffit pour cela de remplacer $\varepsilon$ par $\frac{1}{\omega(\varepsilon)} \varepsilon$ ). En remplaçant dans la relation $\left(^{*}\right) x$ par $\varepsilon$ et $y$ par $\varepsilon^{2}$ on obtient $\left(\varepsilon^{2}\right)^{2}=\omega\left(\varepsilon^{2}\right) \varepsilon^{2}$; ainsi, $e=\frac{1}{\omega\left(\varepsilon^{2}\right)} \varepsilon^{2}$ est un idempotent de $A$ et $\omega(e)=1$. Donc $A=\operatorname{Ke} \oplus \operatorname{Ker}(\omega), \omega(\lambda e+x)=\lambda$ et les relations caractérisant une algèbre BJF résultent de la polarisation de l'identité $(e+x)^{3}=(e+x)^{2}$.

Théorème 2.9. Soit $A$ une algèbre BJF. Les conditions suivantes sont équivalentes:

(a) $S$ est un idéal;

(b) $A$ est pondérée;

(c) $A$ est de Bernstein d'ordre $\leqq 1$;

(d) $A$ est de Jordan.

Démonstration. Si $S$ est un idéal, la forme linéaire $\omega$ associée à $A$ et définie par $\omega(\lambda e+x)=\lambda$ est une pondération d'où $a \Rightarrow b$. Puis, si $A$ admet une pondération $\omega$, elle est identique à la forme linéaire associée: en effet, de 2.3 on obtient $S \subset \operatorname{Ker}(\omega)$; or forcément $\omega(e)=1$ (car $\omega \neq 0$ ) ce qui entraîne $S=\operatorname{Ker}(\omega)$, donc $b \Rightarrow a$; de plus, comme $\omega$ vérifie l'identité $y^{3}=\omega(y) y^{2}$ pour tout $y$ dans $A$ on a $b \Rightarrow c$ et $b \Rightarrow d$ (Exemple 2.7). Que $c \Rightarrow b$ est immédiat. Enfin, montrons que $d \Rightarrow c$ : si $A$ est de Jordan pour tout $x \in A$ on a $x^{2} x^{2}=x\left(x x^{2}\right)=x\left(x^{3}\right)=x\left(\omega(x) x^{2}\right)=\omega(x) x^{3}=\omega(x)^{2} x^{2}$. Or, de $2 t(t z)+t^{2} z=2 \omega(t) t z+$ $\omega(z) t^{2}\left(^{*}\right)$ (voir démonstration 2.8), en remplaçant $t$ par $x$ et $z$ par $x^{2}$, on obtient $x^{2} x^{2}=\omega\left(x^{2}\right) x^{2}$. Si $x^{2} \neq 0$ on a forcément $\omega\left(x^{2}\right)=\omega(x)^{2}$. Si $x^{2}=0$, en remplaçant dans $\left(^{*}\right) t$ par $x$ et $z$ par $e$ on a $2 x(e x)=2 \omega(x) e x$; or, $2 x(e x)=x^{2}-e x^{2}$ (rel. 2.2) donc $\omega(x) e x=0$. Si $e x=0$ en remplçant dans $\left(^{*}\right) t$ par $e$ et $z$ par $x$ on obtient $\omega(x) e=0$ d'où $\omega(x)=0$. On en conclut que $\omega\left(x^{2}\right)=\omega(x)^{2}$ pour tout $x$ dans $A$, donc $\omega$ est multiplicative et $A$ est de Bernstein.

\section{Structure de l'algèbre}

Proposition 3.1. Soit $x \in A$. Alors $x \in S \Leftrightarrow e x \in S ; e x=\frac{1}{2} x \Rightarrow x \in S ; e x=0 \Rightarrow x \in S$.

Démonstration. Pour la première affirmation, si $x \in S$, on pose $e x=\lambda e+z$; par 2.1 on a $\lambda e+z=2 \lambda e+2 e z$ d'ou $\lambda e=z-2 e z=e(z-2 e z)=0$ car $z \in S$. La réciproque est immédiate. Pour les deux dernières, on pose $x=\lambda e+z$ avec $\lambda \in K$ et $z \in S$ et on applique la relation 2.1 .

Soient $U=\left\{x \in S / e x=\frac{1}{2} x\right\}$ et $V=\{x \in S / e x=0\}$. On a:

Proposition 3.2. On a: $S=U \oplus V, U^{2} \subset V, U V \subset U$ et $V^{2} \subset K e$.

Démonstration. Il est clair que $U \cap V=\{0\}$. De plus, $x=2 e x+(x-2 e x)$ pour tout $x \in S$ ce qui montre la première assertion. Pour le reste, $U^{2} \subset V$ et $U V \subset U$ résultent directement des relations 2.2 et 2.4. Montrons $V^{2} \subset \mathrm{Ke}$ : soit $x \in V$; on pose $x^{2}=$ 
$\lambda e+u+v$ avec $\lambda \in K, u \in U$ et $v \in V$. Comme $e x=0$, on a $x^{2}=e x^{2}(2.2)$, d'où $\lambda e+u+v=$ $\lambda e+\frac{1}{2} u$, donc $u=v=0$.

La somme directe $\mathrm{Ke} \oplus U \oplus V$ s'appelle décomposition de Peirce de $A$ par rapport à l'idempotent $e$.

Corollaire 3.3. Soient $u, u^{\prime}$ dans $U$ et $v, v^{\prime}$ dans $V$. On a les identités suivantes: $\left(u u^{\prime}\right) v=0, u\left(u^{\prime} v\right)+u^{\prime}(u v)=0$ et $v\left(v^{\prime} u\right)+v^{\prime}(v u)+\left(v v^{\prime}\right) u=0$.

Démonstration. En effet, soient $u, u^{\prime}$ deux éléments de $U$ et $v$ un élément de $V$. De la relation 2.5 on obtient $\left(u u^{\prime}\right) v+u\left(u^{\prime} v\right)+u^{\prime}(u v)=0$; d'après la proposition 3.2 on sait que $\left(u u^{\prime}\right) v$ est dans $\mathrm{Ke}$ et que $u\left(u^{\prime} v\right)+u^{\prime}(u v)$ est un élément de $V$ d'où le résultant. Pour le reste, il suffit d'appliquer la relation 2.5 .

On pose $V_{\perp}=\operatorname{Ann}_{v}(V)=\{v \in V / v V=\{0\}\}$.

Il est clair que si $V_{\perp}=V$, l'algèbre est de Bernstein-Jordan (Thm. 2.9).

On a le résultat suivant:

Proposition 3.4. Tout supplémentaire non trivial de $V_{\perp}$ par rapport à $V$ admet une base de carrés non nuls.

Démonstration. Si $V-V_{\perp} \neq\{0\}$ il existe $v, v^{\prime} \in V-V_{\perp}$ tels que $v v^{\prime} \neq 0$; alors les carrés de $v$, $v^{\prime}$ et $v+v^{\prime}$ ne peuvant pas être tous nuls. Soit donc $V_{e}$ un sous-espace supplémentaire de $V_{\perp}$ par rapport à $V$ et $L=\left\{v_{1}, v_{2}, \ldots, v_{m}\right\}$ un système libre de $V_{e}$ formé par un nombre maximal d'éléments de carré non nul (on sait que $m \geqq 1$ ). Le sous-espace engendré par $L$ est $V_{e}$ : en effet, dans le cas contraire, soit $v \in V_{e}$ tel que le système $\left\{v, v_{1}\right.$, $\left.v_{2}, \ldots, v_{m}\right\}$ est libre, or pour tout $\lambda \in K$ et $v_{i} \in L$ on a forcément $\left(v_{i}+\lambda v\right)^{2}=0$ (sinon, on remplacerait $v$ par un des $v_{i}+\lambda v$ ce qui contredirait la maximalité de $m$ ) d'où, en prenant trois valeurs convenables de $\lambda$, on obtient, $v_{i}^{2}=0$. Contradiction.

Dorénavant, $V_{e}$ désigne un supplémentaire de $V_{\perp}$ par rapport à $V$.

Pour tout $v \in V$ on note $v^{2}=\alpha_{v} e$ avec $\alpha_{v}$ dans $K$.

Proposition 3.5. On a l'inclusion $U^{2} \subset V_{\perp}$ et pour tout $u \in U$, $v \in V$ et $v^{\prime} \in V_{\perp}$ on a les identités suivantes: $v(u v)=-\frac{1}{4} \alpha_{v} u, v^{\prime}\left(u v^{\prime}\right)=0, v\left(v^{\prime} u\right)+v^{\prime}(v u)=0,(u v)^{2}=\frac{1}{4} \alpha_{v} u^{2},\left(u v^{\prime}\right)^{2}=0$ et $(u v)\left(u v^{\prime}\right)=0$.

Démonstration. Montrons que $(u v)^{2}=\frac{1}{4} \alpha_{v} u^{2}$. En effet, par la proposition 3.2 et le Corollaire 3.3 on a $(u v)^{2}=-u((u v) v)$; mais, encore par le Corollaire 3.3, $(u v) v=-\frac{1}{4} \alpha_{v} u$, d'où le résultat. 
Proposition 3.6. Si $U \neq\{0\}$ on a:

(a) $V^{2} \neq\{0\} \Leftrightarrow$ il existe $v \in V$ tel que $L_{v}: U \rightarrow U, L_{v}(u)=v u$ est un automorphisme despaces vectoriels. Dans ces conditions, $U V=U$.

(b) Soit $v \in V$. Si $v U=\{0\}$ alors $v \in \operatorname{Ann}_{A}(A)$.

Démonstration. Si $V^{2} \neq\{0\}, V \neq V_{1}$. Soit $v \in V$ tel que $v^{2} \neq 0$; comme la dimension de $U$ est finie il suffit de prouver que l'application $L_{v}: U \rightarrow U$ est injective, ce qui découle de l'identité $(u v) v=-\frac{1}{2} v^{2} u$ de la proposition précédente. Réciproquement, s'il existe $v \in V$ tel que $L_{v}: U \rightarrow U$ est bijectif on a $u \neq 0 \Rightarrow L_{v}(u) \neq 0 \Rightarrow L_{v}(u v)=v(u v) \neq 0 \Rightarrow v^{2} \neq 0$ (3.5). Quant à (b), soit $v^{\prime} \in V$ et $u \neq 0$; on a $u\left(v v^{\prime}\right)+v^{\prime}(u v)+v\left(v^{\prime} u\right)=0$ (3.3) d'où $u\left(v v^{\prime}\right)=0$ (3.2) et forcément $v v^{\prime}=0$ ce qui prouve que $v \in V_{\perp}$ d'où le résultat.

Considérons l'algèbre $\mathrm{BJF}$ de base $\left\{e, u, v, v^{\prime}\right\}$ et sa table de multiplication: $e^{2}=e$, $e u=\frac{1}{2} u, u v=\frac{1}{2} u, v^{2}=-e$, les autres produit étant nuls. Elle est isomorphe à celle de base $\{e, u, v, w\}$ et de table de multiplication: $e^{2}=e, e u=u, u v=\frac{1}{2} u, u w=\frac{1}{2} u, v^{2}=-e, w^{2}=-e$, $v w=-e$, les autres produites étant nuls (il suffit de poser $w=v-v^{\prime}$ ).

Il est clair que si $\lambda \neq 0$, il existe $v \in V$ tel que $v^{2}=-\lambda^{2} e$ si et seulement si il existe $v \in V$ tel que $v^{2}=-e$.

On verra que ces éléments de $V$ jouent un rôle important dans la structure des espaces $\operatorname{Ann}_{A}(u), u \in U$, et dans celle des invariants des transformations de Peirce.

Théorème 3.7. Si $U \neq\{0\}$ et $\lambda \neq 0$ est un élément de $K$, il existe $v \in V$ tel que $v^{2}=-\lambda^{2} e$ si et seulement si il existe $u \in U, u \neq 0$, tel que $u v= \pm \frac{1}{2} \lambda u$.

Démonstration. Dans ce qui suit, les identités sont justifiées par les résultats de la Proposition 3.5. Soit $u \in U-\{0\}$; on sait que $u v \neq 0$ (Proposition 3.6). Si $u v \neq \pm \frac{1}{2} \lambda u$ on pose $u v= \pm \frac{1}{2} \lambda u+u^{\prime}$ avec $u^{\prime} \in U-\{0\}$; en multipliant par $v$ on obtient $\frac{1}{4} \lambda^{2} u=(u v) v=$ $\pm \frac{1}{2} \lambda u v+u^{\prime} v= \pm \frac{1}{2} \lambda\left( \pm \frac{1}{2} \lambda u+u^{\prime}\right)+u^{\prime} v$; il en résulte $u^{\prime} v= \pm \frac{1}{2} \lambda u^{\prime}$. Réciproquement, si $u v=$ $\pm \frac{1}{2} \lambda u$, on obtient $v(u v)=\frac{1}{4} \lambda^{2} u$ d'où $v^{2}=-\lambda^{2} e$.

Si $\sigma \in U, \operatorname{Ann}_{s}(\sigma)=\operatorname{Ann}_{U}(\sigma) \oplus \operatorname{Ann}_{V}(\sigma)$ et on montre sans difficulté, à l'aide du Corollaire 3.3 et de la Proposition 3.5, que cet espace est une sous-algèbre et $\operatorname{Ann}_{V}(\sigma)$ une zéro-sous-algèbre.

Soit $\sigma \in U, \sigma \neq 0$. Notons $0_{\sigma}$ le sous-espace engendré par les éléments $\lambda e+v$ avec $v \in V$, $\sigma v=\frac{1}{2} \lambda \sigma$ et $0_{\sigma}^{-}$le sous-espace engendré par les éléments $\lambda e+v$ avec $v \in V, \sigma v=-\frac{1}{2} \lambda \sigma$. On remarque que $\lambda e+v$ est dans $0_{\sigma}$ si et seulement si $\lambda e-v$ est dans $0_{\sigma}^{-}$. De plus:

Proposition 3.8. Les sous-espaces $0_{\sigma}, 0_{\sigma}^{-}$sont des zéro-sous-algèbres. De plus, $0_{\sigma} \cap 0_{\sigma}^{-}=\operatorname{Ann}_{V}(\sigma)$ et si $\sigma^{2} \neq 0$ alors $0_{\sigma}=0_{\sigma}^{-}$.

Démonstration. Montrons que $0_{\sigma}$ est une zéro-algèbre: soient $\lambda e+v$ et $\lambda^{\prime} e+v^{\prime}$ dans $0_{a}$; on a $\sigma\left(v+v^{\prime}\right)=\frac{1}{2}\left(\lambda+\lambda^{\prime}\right) \sigma$ d'où, par $3.7,\left(v+v^{\prime}\right)^{2}=-\left(\lambda+\lambda^{\prime}\right)^{2} e$; mais comme $v^{2}=-\lambda^{2} e$ et $v^{\prime 2}=-\lambda^{\prime 2} e$ on en déduit que $v v^{\prime}=-\lambda \lambda^{\prime} e$. Il s'ensuit que $(\lambda e+v)\left(\lambda^{\prime} e+v^{\prime}\right)=$ 
0 . Pour ce qui est de la deuxième affirmation, si $\lambda e+v=\lambda^{\prime} e-v^{\prime}$ alors $\lambda=\lambda^{\prime}$ et $v=-v^{\prime}$, d'où $-\frac{1}{2} \lambda \sigma=\sigma v=-\sigma v^{\prime}=\frac{1}{2} \lambda \sigma$ donc $\lambda=0$ et $v \in \operatorname{Ann}_{V}(\sigma)$; la réciproque est immédiate. La dernière affirmation découle de la Proposition 3.5.

Théorème 3.9. $A \operatorname{Ann}_{A}(\sigma)=\mathrm{Ann}_{U}(\sigma) \oplus 0_{\sigma}^{-}$et $\mathrm{Ann}_{A}(\sigma)$ est une sous-algèbre vérifiant $\operatorname{Ann}_{A}(\sigma)^{2} \subset \operatorname{Ann}_{S}(\sigma)$.

Démonstration. Soit $x=\lambda e+u+v$. Si $\sigma x=0$ on a $\sigma u=0$ et $\frac{\lambda}{2} \sigma+\sigma v=0$; il s'ensuit que l'élément $\lambda e+v$ est dands $0_{\sigma}^{-}$. La réciproque se fait sans difficulté. Enfin, de la Proposition 3.2 et du Corollaire 3.3 résulte $\operatorname{Ann}_{U}(\sigma)^{2} \subset \operatorname{Ann}_{V}(\sigma)$; puis, si $\lambda e+v$ est dans $0_{\sigma}^{-}$et $u$ dans $U$ on a: $\sigma(u(\lambda e+v))=\frac{\lambda}{2} \sigma u+\sigma(u v)=\frac{\lambda}{2} \sigma u-u(\sigma v)=\lambda \sigma u$ (Corollaire 3.3), d'où si $u$ est un élément de $\operatorname{Ann}_{U}(\sigma)$ on obtient $\sigma(u(\lambda e+v))=0$, d'où $\operatorname{Ann}_{U}(\sigma) 0_{\sigma}^{-} \subset \operatorname{Ann}_{S}(\sigma)$. Ainsi $\operatorname{Ann}_{A}(\sigma)$ est une sous-algèbre car $\operatorname{Ann}_{U}(\sigma)$ et $0_{\sigma}^{-}$sont des zéro-algèbres.

Proposition 3.10. Soient $x \neq 0$ un élément de $0_{\sigma}$ et $L_{x}: U \rightarrow U, L_{x}(u)=x u$. Alors $U=\operatorname{Ker}\left(L_{x}\right) \oplus \operatorname{Im}\left(L_{x}\right), \operatorname{Ker}\left(L_{x}\right)=\left\{u \in U / x \in 0_{u}^{-}\right\}$et $\operatorname{Im}\left(L_{x}\right)=\operatorname{Ann}_{U}(\sigma)$.

Démonstration. Si $x=\lambda e+v$ est un élément de $0_{\sigma}$ on sait que $v u=\frac{\lambda}{2} u$; ainsi $L_{x}(u)=x u=\lambda u$ d'où $L_{x}^{2}=\lambda L_{x}$; or, comme $x \neq 0, \lambda \neq 0$, et l'application linéaire $\frac{1}{\lambda} L_{x}$ est un projecteur d'où $U=\operatorname{Ker}\left(L_{x}\right) \oplus \operatorname{Im}\left(L_{x}\right)$. Pour le reste, on a $L_{x}(u) \sigma=\frac{\lambda}{2} u \sigma+(u v) \sigma=$ $\frac{\lambda}{2} u \sigma-(\sigma v) u=\frac{1}{2} u \sigma-\frac{\lambda}{2} u \sigma=0$ d'où $\operatorname{Im}\left(L_{x}\right) \subset \operatorname{Ann}_{v}(\sigma)$; puis, si $u^{\prime}$ est un élément de $A n_{U}(\sigma)$, $L_{x}\left(\frac{1}{\lambda} u^{\prime}-\frac{2}{\lambda^{2}} u^{\prime} v\right)=u^{\prime}$ car $v\left(u^{\prime} v\right)=\frac{\lambda^{2}}{4} u^{\prime}$ ce qui montre que $\operatorname{Ann}_{U}(\sigma) \subset \operatorname{Im}\left(L_{x}\right)$. L'égalité $\operatorname{Ker}\left(L_{x}\right)=\left\{u \in U / x \in 0_{u}^{-}\right\}$s'établit sans problèmes.

\section{Les transformations de Peirce}

Soit $\operatorname{Ip}(A)$ l'ensemble des idempotents non nuls de $A$.

Proposition 4.1. L'application $U \rightarrow \operatorname{Ip}(A)$ définie par $\sigma \rightarrow e+\sigma+\sigma^{2}$ est bijective.

Démonstration. Montrons la surjectivité: soit $x=\lambda e+u+v$ un idempotent non nul de $A$; comme $(\lambda e+u+v)^{2}=\lambda e+u+v$ de 3.2 nous obtenons les équations suivantes: $\lambda^{2}+\alpha_{v}=\lambda, \lambda u+2 u v=u$ et $u^{2}=v$. Or, de 3.3 on déduite que $u v=0$ d'où $\alpha_{v}=0$ et $\lambda=1$ car sinon $v=2 u(u v)=0$, a fortiori $u=0$ et $x=0$, ce qui contredit l'hypothèse. Il s'ensuit que $x=e+u+u^{2}$.

Soit $\sigma \in U$. On note $e_{\sigma}=e+\sigma+\sigma^{2}$ et $\mathrm{Ke}_{\sigma} \oplus U_{\sigma} \oplus V_{\sigma}$ la décomposition de Peirce de $A$ par rapport à $e_{\sigma}$.

On appelle invariant de structure tout résultat concernant l'algèbre $A$ ne dépendant pas du choix de la décomposition de Peirce.

Proposition 4.2. On $a$ : $U_{\sigma}=\{u+2 \sigma u / u \in U\}$ et $V_{\sigma}=\{v-2 \sigma v / v \in V\}$.

Démonstration. Soit $x=\lambda e+u+v$ un élément de $A$. Si $e_{\sigma} x=\frac{1}{2} x$, de la définition de $U$ et $V$, de la Proposition 3.2 et du Corollaire 3.5 , on obtient les équations $\frac{1}{2} \lambda e=0$, 
$\frac{1}{2} \lambda \sigma+u \sigma^{2}+v \sigma=0$ et $\frac{1}{2} v=\sigma u$ dont on déduit que $x=u+2 \sigma u$. De même, si $e_{\sigma} x=0$, alors $\lambda e=0, \frac{1}{2} \lambda \sigma+\frac{1}{2} u+u \sigma^{2}+v \sigma=0$ et $\sigma u=0$; il s'ensuit que $u \sigma^{2}=0$ (2.5) et $x=v-2 \sigma v$.

On pose $u_{\sigma}=u+2 \sigma u, u \in U$ et $v_{\sigma}=v-2 \sigma v, v \in V$.

On appelle transformation de Peirce l'application $\phi_{\sigma}: A \rightarrow A$ définie par $\phi_{\sigma}=I+$ $4 L_{\sigma} L_{e}-4 L_{e} L_{\sigma}+4\left(L_{\sigma} L_{e}\right)^{2}$, où $l$ est l'application identité et $L_{x}$ le produit par $x$ et on note $P_{e}$ l'ensemble $\left\{\phi_{\sigma} / \sigma \in U\right\}$. On a:

Théorème 4.3. $\phi_{\sigma}$ est un automorphisme d'espaces vectoriels vérifiant $\phi_{\sigma}(\lambda e+u+v)=$ $\lambda e_{\sigma}+u_{\sigma}+v_{\sigma}, \phi_{\sigma}\left(V_{\perp}\right)=\left(V_{\sigma}\right)_{\perp}, \phi_{\sigma}\left(V_{e}\right)=V e_{\sigma}$ et $\phi_{\sigma}(\operatorname{Ip}(A))=\operatorname{Ip}(A)$.

Démonstration. Que $\phi_{\sigma}(\lambda e+u+v)=\lambda e_{\sigma}+u_{\sigma}+v_{\sigma}$ et que $\phi_{\sigma}$ est un automorphisme d'espaces vectoriels est immédiat. Montrons que $\phi_{\sigma}\left(V_{\perp}\right)=\left(V_{\sigma}\right)_{\perp}$ : si $v \in V_{\perp}$ et $v^{\prime} \in V$ on a $(v-2 \sigma v)\left(v^{\prime}-2 \sigma v^{\prime}\right)=-2\left[(\sigma v) v^{\prime}+\left(\sigma v^{\prime}\right) v-2(\sigma v)\left(\sigma v^{\prime}\right)\right]=0$ (Corollaire 3.5), ce qui montre que $v_{\sigma} \in\left(V_{\sigma}\right)_{\perp}$. La réciproque résulte du fait que $V^{2} \subset \mathrm{Ke}$. Avec les mêmes techniques on montre que $\phi_{\sigma}\left(V_{e}\right)=V e_{\sigma}$. Pour ce qui est de la dernière assertion, il suffit de remarquer que $\phi_{\sigma}\left(e_{\tau}\right)=e_{u}, u=\tau+\sigma-2 \sigma \tau^{2}$.

Il en résulte que les dimensions des sous-espaces $U, V, V_{\perp}$ et $V_{\mathrm{e}}$ sont des invariants de structure. On peut $y$ ajouter les résultats suivants:

Proposition 4.4. Les sous-espaces $U V \oplus U^{2}, V^{2}, \operatorname{Ann}_{v}(U), \operatorname{Ann}_{V}(V)$ et $\operatorname{Ann}_{V}(U)$ ainsi que les dimensions de $U V, U^{2}$ et $V^{2}$ sont des invariants de structure. Il en est de même des situations $U^{3}=\{0\}, U(U V)=\{0\}, U^{2}(U V)=\{0\}$ et $U V=\{0\}$.

Démonstration. De la Proposition 3.2 on obtier:t que $S^{2}=V^{2} \oplus U V \oplus U^{2}$; ceci est indépendant de la décomposition de Peirce choisie. De plus, toute transformation de Peirce vérifie $\phi_{\sigma}\left(u^{2}\right)=\phi_{\sigma}(u)^{2}$ et $\phi_{\sigma}\left(v^{2}\right)=\phi_{\sigma}(v)^{2}$ pour tout $u \in U$ et $v \in V$, ce qui entraîne que les dimensions de $U^{2}$ et $V^{2}$ et, a fortiori, celle de $U V$ sont des invariants de structure. Soient $\theta \in \operatorname{Ann}_{U}(U)$ et $u_{\sigma} \in U_{\sigma}$; or $\theta \in U_{\sigma}$ car $\phi_{\sigma}(\theta)=\theta$ et $\theta u_{\sigma}=\theta(u+2 \sigma u)=2 \theta(\sigma u)=0$ (relation 2.5), c'est-à-dire que $\theta \in \operatorname{Ann}_{U_{\sigma}}\left(U_{\sigma}\right)$. On montre de même que $\operatorname{Ann}_{U}(V)$ et $\operatorname{Ann}_{V}(U)$ sont aussi des invariants de structure. Montrons que la situation $U^{3}=\{0\}$ ne dépend pas de la décomposition de Peirce choisie: en effet, soient, $x, y, z \in U$; on a $x_{\sigma}\left(y_{\sigma} z_{\sigma}\right)=(x+2 \sigma x)[(y+2 \sigma y)(z+2 \sigma z)]=(x+2 \sigma x)(y z+2 y(\sigma z)+2 z(\sigma y)+4(\sigma y)(\sigma z)) . \quad$ Or $U^{2} \subset V_{\perp}$ donc $\left(U^{2}\right)^{2}=\{0\}$; ainsi, si $U^{3}=\{0\}$ il en résulte que $x_{\sigma}\left(y_{\sigma} z_{\sigma}\right)=0$, c'est-à-dire, $U_{\sigma}^{3}=\{0\}$. Enfin, soient $x, y \in U$ et $z \in V$; si $U(U V)=\{0\}, x_{\sigma}\left(y_{\sigma} z_{\sigma}\right)=(x+2 \sigma x)[(y+2 \sigma y)$ $(z-2 \sigma z)]=2(\sigma x)(y z)-8(\sigma x)[(\sigma y)(\sigma z)]$. Mais de la relation 2.5 on a $(\sigma x)(y z)+\sigma(x(y z))+$ $x(\sigma(y z))=0$ d'où $(\sigma x)(y z)=0$ car $x(y z)=\sigma(y z)=0$ par hypothèse; de la méme façon on montre que $(\sigma x)[(\sigma y)(\sigma z)]=0$. Il s'ensuit que $U_{\sigma}\left(U_{\sigma} V_{\sigma}\right)=\{0\}$. Avec les mêmes techniques on montre que les situations $U^{2}(U V)=\{0\}$ et $U V=\{0\}$ sont aussi des invariants de structure. 
Soit $\operatorname{Inv}\left(\phi_{\sigma}\right)$ le sous-espace des points fixes de $\phi_{\sigma}$. On montre sans difficulté que $\operatorname{Inv}\left(\phi_{\sigma}\right) \cap S=\operatorname{Ann}_{S}(\sigma)$. Plus précisement,

Proposition 4.5. On a: $\operatorname{Inv}\left(\phi_{\sigma}\right)=\mathrm{Ann}_{U}(\sigma) \oplus 0_{\sigma}$ et $\operatorname{Inv}\left(\phi_{\sigma}\right)$ est une sous-algèbre vérifiant $\operatorname{Inv}\left(\phi_{\sigma}\right)^{2} \subset \operatorname{Ann}_{S}(\sigma)$.

Démonstration. Soit $x=\lambda e+u+v$ et $x^{-}=\lambda e+u-v$. On a $\phi_{\sigma}(x)=x$ si et seulement si $\sigma x^{-}=0$. Ainsi, le résultat découle du Théorème 3.9.

Corollaire 4.6. Les sous-espaces $\operatorname{Inv}\left(\phi_{\sigma}\right)$ et $\operatorname{Ann}_{A}(\sigma)$ sont canoniquement isomorphes par l'application linéaire involutive $x \rightarrow x^{-}$, isomorphisme d'algèbres si et seulement si $\operatorname{Inv}\left(\phi_{\sigma}\right)=\operatorname{Ann}_{A}(\sigma)$ ou $\operatorname{Inv}\left(\phi_{\sigma}\right) \cap \operatorname{Ann}_{A}(\sigma)=\{0\}$.

Démonstration. La première affirmation découle du Théorème 3.9 et de la Proposition 4.5. La deuxième, de l'équation $x^{2}=x^{-2}$ et de la Proposition $3.6 \operatorname{car} \operatorname{Inv}\left(\phi_{\sigma}\right)=$ $\operatorname{Ann}_{A}(\sigma) \Leftrightarrow 0_{\sigma}=\{0\}$ et $\operatorname{Inv}\left(\phi_{\sigma}\right) \cap \operatorname{Ann}_{A}(\sigma)=\{0\} \Leftrightarrow \operatorname{Ann}_{U}(\sigma)=\{0\}$.

\section{A propos des automorphismes}

La caractérisation des automorphismes des algèbres de Bernstein est encore un problème ouvert; sauf dans des cas très particuliers, on ne connaît pas leur forme et moins encore la structure du groupe qu'ils conforment.

Dans ce qui suit, nous abordons certains aspects du problème.

Proposition 5.1. Soient $A=\operatorname{Ke} \oplus S$ une algèbre BJF et $\phi: A \rightarrow A$ un automorphisme. Alors $\phi(e)=e_{\tau} \in \operatorname{Ip}(A), \phi(U)=U_{\tau}, \phi(V)=V_{\tau}, \phi\left(V_{\perp}\right)=\left(V_{\tau}\right)_{\perp}$ et $\phi\left(V_{e}\right)=V_{e_{\tau}}$.

Démonstration. De $\phi(e)^{2}=\phi\left(e^{2}\right)=\phi(e)$, on déduit que $\phi(e)$ est dans $\operatorname{Ip}(A)$. Soit $\phi(e)=e_{\tau}=e+\tau+\tau^{2}$ avec $\tau \in U$. Montrons par exemple que $\phi(V)=V_{\tau}$ : soit $v^{\prime} \in V$ et posons $\phi\left(v^{\prime}\right)=\lambda e+u+v$; on a $0=\phi\left(e v^{\prime}\right)=e_{\tau} \phi\left(v^{\prime}\right)$ d'où par 3.2 et $3.5, \lambda=0$ et $u=-2 \tau v$. Ainsi, $\phi\left(v^{\prime}\right)=v-2 \tau v$.

Proposition 5.2. La transformation de Peirce $\phi_{\sigma}$ est un automorphisme d'algèbres si et seulement si $\sigma^{2}(U V)=\{0\}$.

Démonstration. Soient $u \in U$ et $v \in V$. Du Corollaire 3.3 et de la Proposition 3.5 on a: $\phi_{\sigma}(u)^{2}=(u+2 \sigma u)^{2}=u^{2}+4 u(\sigma u)=u^{2}-2 \sigma u^{2}=\phi_{\sigma}\left(u^{2}\right)$ et $\phi_{\sigma}(v)^{2}=(v+2 \sigma v)^{2}=v^{2}+4 v(\sigma v)+$ $4(\sigma v)^{2}=\alpha_{v} e+\alpha_{v} \sigma+\alpha_{v} \sigma^{2}=\alpha_{v}\left(e+\sigma+\sigma^{2}\right)=\alpha_{v} \phi_{\sigma}(e)=\phi_{\sigma}\left(\alpha_{v} e\right)=\phi_{\sigma}\left(v^{2}\right)$, ceci sans recours à l'hypothèse. Or, $\phi_{\sigma}(u) \phi_{\sigma}(v)=u v+2(\sigma u) v-2 u(\sigma v)-4(\sigma u)(\sigma v)$; mais $\sigma u \in V_{\perp}$, d'où, par 3.3 et $3.5, \phi_{\sigma}(u) \phi_{\sigma}(v)=u v+2 \sigma(u v)+2 \sigma^{2}(u v)$. D'autre côté, $\phi_{\sigma}(u v)=u v+2 \sigma(u v)$; donc $\phi_{\sigma}(u v)=$ $\phi_{\sigma}(u) \phi_{\sigma}(v)$ si et seulement si $\sigma^{2}(U V)=\{0\}$.

Proposition 5.3. Si $\sigma^{2} U=\{0\}$, alors $\phi_{\sigma}$ est un automorphisme d'algèbres et $\operatorname{dim}_{K}\left(\operatorname{Inv}\left(\phi_{\sigma}\right)\right) \geqq \frac{1}{2}\left[\operatorname{dim}_{K}(A)-1\right]$. 
Démonstration. La première affirmation découle trivialement de 5.2. Pour la deuxième, soit $\psi$ la restriction de $\phi_{\sigma}-l$ sur $S$; il suffit de voir que $\sigma^{2} U=0$ si et seulement si $\psi^{2}=0$. Dans ce cas, $\operatorname{Im}(\psi) \subset \operatorname{Ker}(\psi)$ d'où la conclusion.

Théorème 5.4. Soient $\tau$ et $\sigma$ deux éléments de $U$. Alors $\phi_{\sigma}$ est un automorphisme d'algèbres si et seulement s'il en est de même de $\phi_{\sigma_{r}}$.

Démonstration. Il s'agit de montrer que $\sigma_{\tau}^{2}\left(u_{\tau} v_{\tau}\right)=0$ si et seulement si $\sigma^{2}(u v)=0$. Soient $u \in U$ et $v \in V$; on a: $\sigma_{\tau}^{2}\left(u_{\tau} v_{\tau}\right)=(\sigma+2 \tau \sigma)^{2}((u+2 \tau u)(v-2 \tau v))$; or $(\sigma+2 \tau \sigma)^{2}=\sigma^{2}-2 \tau \sigma^{2}$ car $2 \sigma(\tau \sigma)=-2 \tau \sigma^{2}$ (Proposition 3.3) et $(\tau \sigma)^{2}=0$ (Proposition 3.5); de même, on obtient $(u+2 \tau u)(v-2 \tau v)=u v+2 \tau(u v)+2 \tau^{2}(u v)$. Ainsi $\sigma_{\tau}^{2}\left(u_{\tau} v_{\tau}\right)=\sigma^{2}(u v)-2\left(\tau \sigma^{2}\right)(u v)+2 \sigma^{2}(\tau(u v))-$ $4\left(\tau \sigma^{2}\right)(\tau(u v))+2 \sigma^{2}\left(\tau^{2}(u v)\right)-4\left(\tau \sigma^{2}\right)\left(\tau^{2}(u v)\right)$.

Des Propositions $3.2,3.3$ et 3.5 nous obtenons $\left(\tau \sigma^{2}\right)(u v)=-\tau\left(\sigma^{2}(u v)\right), \sigma^{2}(\tau(u v))=0$, $\left(\tau \sigma^{2}\right)(\tau(u v))=-(u v)\left(\tau\left(\tau \sigma^{2}\right)\right)=0, \quad \sigma^{2}\left(\tau^{2}(u v)\right)=-\tau^{2}\left(\sigma^{2}(u v)\right) \quad$ et $\quad\left(\tau \sigma^{2}\right)\left(\tau^{2}(u v)\right)=$ $-(u v)\left(\tau^{2}\left(\tau \sigma^{2}\right)\right)=(u v)\left(\sigma^{2} \tau^{3}\right)=0$ car $\tau^{3}=0$.

Donc, $\sigma_{\tau}^{2}\left(u_{\tau} v_{\tau}\right)=\sigma^{2}(u v)-2 \tau^{2}\left(\sigma^{2}(u v)\right)+2 \tau\left(\sigma^{2}(u v)\right)=\sigma^{2}(u v)+2\left(\tau-\tau^{2}\right)\left(\sigma^{2}(u v)\right)$.

Il est clair que $\sigma^{2}(u v)=0$ entraîne $\sigma_{\tau}^{2}\left(u_{\tau} v_{\tau}\right)=0$. Réciproquement, comme $\sigma^{2}(u v)$ et $2 \tau^{2}\left(\sigma^{2}(u v)\right)$ sont des éléments de $U$ et $\tau\left(\sigma^{2}(u v)\right.$ ) est dans $V$ (Proposition 3.2) si $\sigma_{\tau}^{2}\left(u_{\tau} v_{\tau}\right)=0$, alors $\sigma^{2}(u v)-2 \tau^{2}\left(\sigma^{2}(u v)\right)=0$ et $\tau\left(\sigma^{2}(u v)\right)=0$; or par la Proposition 3.3 on a $\tau^{2}\left(\sigma^{2}(u v)\right)=-2 \tau\left(\tau\left(\sigma^{2}(u v)\right)\right)$, donc $\tau^{2}\left(\sigma^{2}(u v)\right)=0$; ainsi $\sigma^{2}(u v)=0$.

Sous certaines conditions les applications $\phi_{\sigma}^{-1}$ et $\phi_{\theta} \circ \theta_{\sigma}$ sont des transformations de Peirce. Plus précisément:

Proposition 5.5. Soit $\phi_{\sigma}$ un élément de $P_{e}$. Les conditions suivantes sont équivalentes:

(a) $\phi_{\sigma}^{-1} \in P_{e}$;

(b) $\sigma^{2} U=\{0\}$;

(c) $\phi_{\sigma}^{-1}=\phi_{-\sigma}$.

Dans ces conditions $\phi_{\sigma}$ est un automorphisme d'algèbres.

Démonstration. Que $c \Rightarrow a$ est immédiat et l'implication $b \Rightarrow c$ s'établit sans difficulté. Puis, si $\phi_{\sigma}^{-1}=\phi_{\theta}$, de $\left(\phi_{\theta} \circ \phi_{\sigma}\right)(e)=e$ on obtient $\theta+\sigma-2 \theta \sigma^{2}=0$ (3.2) d'où $\theta^{2}=-\theta_{\sigma}$ donc $\theta \sigma^{2}=0$ (3.3); il en résulte $\theta=-\sigma$ (donc $\left.a \Rightarrow c\right)$. Soit $u \in U$, de $\left(\phi_{-\sigma} \circ \phi_{\sigma}\right)(u)=u$ on obtient $\sigma(\sigma u)=0$, c'est-à-dire $\sigma^{2} u=0(3.3)$ donc $a \Rightarrow b$. La dernière affirmation résulte du fait que $U V \subset U$ et de la Proposition 5.2.

Proposition 5.6. Soient $\theta$ et $\sigma$ des éléments de $U$. Les conditions suivantes sont équivalents:

(a) $\phi_{\theta} \circ \phi_{\sigma}$ est une transformation de Peirce;

(b) $\theta(\sigma U)=\theta(\sigma V)=\{0\}$.

Et sous ces conditions $\phi_{\theta} \circ \phi_{\sigma}=\phi_{\theta+\sigma}$. 
Démonstration. Si $\left(\phi_{\theta} \circ \phi_{\sigma}\right)(e)=\phi_{\tau}$ alors $\tau=\theta+\sigma-2 \theta \sigma^{2}$. De $\left(\phi_{\theta} \circ \phi_{\sigma}\right)(u)=\phi_{\mathrm{t}}(u)$ on déduit $\theta(\sigma U)=\{0\}$ d'où $\tau=\theta+\sigma$; enfin, de $\left(\phi_{\theta} \circ \phi_{\sigma}\right)(v)=\phi_{\tau}(v)$ on déduit $\theta(\sigma V)=\{0\}$. La réciproque s'établit sans problèmes.

On montre sans difficulté le résultat suivant:

Proposition 5.7. Soient $\theta, \sigma \in U$. Les conditions suivantes sont équivalentes:

(a) $\phi_{\theta} \circ \phi_{\sigma}=\phi_{\sigma} \circ \phi_{\theta}$

(b) $\theta(\sigma u)=\sigma(\theta u), u \in U$ et $\theta(\sigma V)=\{0\}$.

\section{Démonstration.}

Corollaire 5.8. Soit $\sigma$ un élément de $U$. Les conditions suivantes sont équivalentes:

(a) $\sigma U^{2}=U(\sigma U)=\sigma(U V)=\{0\}$;

(b) $\phi_{\sigma}$ commute avec tous les éléments de $P_{e}$;

(c) $\phi_{\theta} \circ \phi_{\sigma}=\phi_{\theta+\sigma}$ pour tout $\theta$ dans $U$.

Dans ces conditions $\phi_{\sigma}$ est un automorphisme d'algèbres.

Démonstration. Les équivalences découlent des propositions précédentes. Enfin, si $\sigma(U V)=\{0\}, \sigma^{2}(U V)=\{0\}$ (3.3) donc $\phi_{\sigma}$ est un automorphisme d'algèbres (5.2).

On appelle centre de $P_{e}$ et on note $Z\left(P_{e}\right)$ l'ensemble des transformations de Peirce vérifiant la condition (b) du corollaire ci-dessus, on note $Z(U)$ le sous-espace de $U$ formé par les éléments vérifiant la condition (a) du même corollaire. Il est clair que $\left(Z\left(P_{e}\right), \circ\right)$ est un groupe commutatif et que l'application $\zeta:(Z(U),+) \rightarrow\left(Z\left(P_{e}\right), \circ\right)$, $\zeta(\sigma) \rightarrow \phi_{\sigma}$, est un isomorphisme de groupes.

Théorème 5.9. Soit $\phi_{\theta}$ un élément de $P_{e}$. Alors $\phi_{\theta}(Z(U))=Z\left(U_{\theta}\right)$ et l'espace $\operatorname{Ann}_{Z(U)} U$ est un invariant de structure vérifiant $\zeta\left(\mathrm{Ann}_{Z(U)} U\right)=\bigcap_{e \in \operatorname{lp}(A)} Z\left(P_{e}\right)$.

Démonstration. Il faut démontrer d'abord que $\sigma \in Z(U) \Leftrightarrow \sigma_{\theta} \in Z\left(U_{\theta}\right)$. On a: $\sigma_{\theta}\left(u_{\theta} u_{\theta}^{\prime}\right)=\sigma\left(u u^{\prime}\right)-2 \sigma\left(\theta\left(u u^{\prime}\right)\right)-4(\sigma \theta)\left(\theta\left(u u^{\prime}\right)\right)$, car $(\sigma \theta)\left(u u^{\prime}\right) \in V_{\perp}^{2}$; de la même façon, $u_{\theta}\left(\sigma_{\theta} u_{\theta}^{\prime}\right)=u\left(\sigma u^{\prime}\right)-2 u\left(\theta\left(\sigma u^{\prime}\right)\right)-4(u \theta)\left(\theta\left(\sigma u^{\prime}\right)\right)$ et, enfin, comme $(\sigma \theta)(\theta(u v)) \in V_{\perp}^{2}, \sigma_{\theta}\left(u_{\theta} v_{\theta}\right)=$ $\sigma(u v)+2(\sigma \theta)(u v)+2 \sigma\left(\theta^{2}(u v)\right)+2 \sigma(\theta(u v))+4(\sigma \theta)\left(\theta^{2}(u v)\right)$. Dans ce qui suit, les identités sont justifiées par les résultats de 3.2, 3.3 et 3.5. L'implication $\sigma \in Z(U) \Rightarrow \sigma_{\theta} \in Z\left(U_{\theta}\right)$ se fait sans grande difficulté. Montrons la réciproque: si $\sigma_{\theta}\left(u_{\theta} u_{\theta}^{\prime}\right)=0$ on a $\sigma\left(u u^{\prime}\right)-$ $4(\sigma \theta)\left(\theta\left(u u^{\prime}\right)\right)=\sigma\left(\theta\left(u u^{\prime}\right)\right)=0$ (la première expression appartient à $U$, la deuxième à $V$ ); or, $(\sigma \theta)\left(\theta\left(u u^{\prime}\right)\right)=-\left(u u^{\prime}\right)(\theta(\sigma \theta))=\frac{1}{2}\left(u u^{\prime}\right)\left(\sigma \theta^{2}\right)=-\frac{1}{2} \theta^{2}\left(\sigma\left(u u^{\prime}\right)\right)$, mais $\sigma\left(u u^{\prime}\right)=4(\sigma \theta)\left(\theta\left(u u^{\prime}\right)\right)$ d'où $(\sigma \theta)\left(\theta\left(u u^{\prime}\right)\right)=-2 \theta^{2}\left((\sigma \theta)\left(\theta\left(u u^{\prime}\right)\right)\right)=2(\sigma \theta)\left(\theta^{2}\left(\theta\left(u u^{\prime}\right)\right)\right)=-2(\sigma \theta)\left(\theta^{3}\left(u u^{\prime}\right)\right)=0$ et ainsi $\sigma\left(u u^{\prime}\right)=0$. Par une démarche analogue on montre que si $u_{\theta}\left(\sigma_{\theta} u_{\theta}^{\prime}\right)=0$ alors $u\left(\sigma u^{\prime}\right)=0$. On a donc prouvé que $\sigma U^{2}=U(\sigma U)=\{0\}$; de ce fait, si $\sigma_{\theta}\left(u_{\theta} v_{\theta}\right)=0$ on obtient $\sigma(u v)=0$ car $\sigma\left(\theta^{2}(u v)\right)=-(u v)\left(\sigma \theta^{2}\right)=0$. Quant à la deuxième partie du théorème, $\sigma \in Z(U) \cap Z\left(U_{\theta}\right)$ si 
et seulement si $\sigma \in Z(U)$ et $\sigma=\sigma_{\theta}$. Mais $\sigma=\sigma_{\theta}$ est équivalente à $\sigma \theta=0$; on en déduit que $\operatorname{Ann}_{Z(U)} U=\bigcap_{\theta_{\in} U} Z\left(U_{\theta}\right)$ ce qui montre que cet espace ne dépend pas de la décomposition choisie. Il s'ensuite que $\zeta\left(\operatorname{Ann}_{Z(U)} U\right)=\bigcap_{e \in \operatorname{Ip}(A)} Z\left(P_{e}\right)$.

Nous finirons ce paragraphe par la. caractérisation des automorphismes d'un certain type d'algèbre BJF.

Les définitions qui suivent sont inspirées de la littérature classique sur les algèbres de Bernstein [10].

On sait que les situations $U(U V)=\{0\}, U^{3}=\{0\}$ et $U^{2}(U V)=\{0\}$ sont des invariants de structure. On dira qu'une algèbre BJF est:

- fortement-orthogonale si $U^{3}=\{0\}$ et $U(U V)=\{0\}$;

-orthogonale si $U^{3}=\{0\}$;

-quasi-orthogonale si $U^{2}(U V)=\{0\}$.

Il est clair que l'orthgonalité forte entraîne l'orthogonalité, laquelle entraîne la quasi-orthogonalité. Si $V^{2} \neq\{0\}$, l'orthogonalité forte est équivalente à la condition $U^{2}=\{0\}$ (Proposition 3.6) et les notions d'orthogonalité et de quasi-orthogonalité coïncident.

Soit $F_{e}=\left\{f \in \operatorname{Aut}_{K}(A) / f(e)=e\right\}$.

Proposition 5.10. On a:

(a) $F_{e}=\operatorname{Aut}_{K}(A)$ si et seulement si $U=\{0\}$;

(b) pour tout $f \in F_{e}$ et $\phi_{\sigma} \in P_{e}$ on a $f \circ \phi_{\sigma}=\phi_{f(\sigma)} \circ f$.

Démonstration. Montrons (a): si $U=\{0\}, A$ n'admet qu'un seul idempotent; forcément $F_{e}=\operatorname{Aut}_{K}(A)$. Réciproquement, montrons que si $U \neq\{0\}$ il existe $\sigma$ élément de $U$, $\sigma \neq 0$, tel que $\sigma^{2} U V=\{0\}$; il s'en suivra, alors, que la transformation de Peirce $\phi_{\sigma}$ est un automorphisme ne fixant pas e. En effet, on sait que $V_{\perp} U \subset V U \subset U$ et $U^{2} \subset V_{\perp}$ (Propositions 3.2 et 3.5) d'où $U^{2}(V U) \subset V_{\perp} U$; donc si $V_{\perp} U=\{0\}$ le résultat est établit; dans le cas contraire, on choisit $v \in V_{\perp}$ et $u \in U$ tels que $\sigma=v u \neq 0$; on sait que $\sigma^{2}=0$ (Proposition 3.5).

Soit $0_{e}$ l'orbite de $e$ sous l'action de $\operatorname{Aut}_{K}(A)$.

Proposition 5.11. Les conditions suivantes sont équivalentes:

(a) $F_{e}$ est un sous-groupe normal;

(b) pour tout $f \in F_{e}$ et $x \in 0_{e}$ on a $f(x)=x$;

Démonstration. Soient $f \in F_{e}$ et $\phi \in \operatorname{Aut}_{K}(A), \phi(e)=e_{\sigma}$. Si (a) est vérifée il existe $g \in F_{e}$ tel que $f \circ \phi=\phi \circ g$, d'où $(f \circ \phi)(e)=(\phi \circ g)(e)$ donc $f\left(e_{\sigma}\right)=e_{\sigma}$. Réciproquement, si (b) est vérifiée on a $\left(\phi^{-1} \circ f \circ \phi\right)(e)=\phi^{-1}\left(f(\phi(e))=\phi^{-1}(\phi(e))\right.$ ce qui prouve que $F_{e}$ est normal. 
Remarque 5.12. Si $\phi, \psi \in \operatorname{Aut}_{K}(A)$ on a $\phi(e)=\psi(e)$ si et seulement si $\psi^{-1} \circ \phi \in F_{e}$; ainsi, l'application $\mathrm{Aut}_{K}(A) \rightarrow \operatorname{Ip}(A), \phi \rightarrow \phi(e)$, se factorise par l'application canonique $\operatorname{Aut}_{K}(A) \rightarrow \operatorname{Aut}_{K}(A) / F_{e}$ et l'application $\operatorname{Aut}_{K}(A) / \mathrm{F}_{\mathrm{e}} \rightarrow \mathrm{Ip}(A)$. Ces trois applications sont compatibles avec l'action $\operatorname{de}^{A u t}{ }_{K}(A) \operatorname{car} A^{A u t}{ }_{K}(A)$ opère sur $A u_{K}(A) / F_{e} \operatorname{par} \phi[\psi]=$ $[\phi \circ \psi],[\psi]$ désignant la classe de $\psi$.

On dira que $A$ est homogène si $\operatorname{Ip}(A)$ est homogène sous $A_{\text {ut }}(A)$, c'est-à-dire, si $\operatorname{Ip}(A)=0_{e}$.

Proposition 5.13. Si $U$ est homogène sous $F_{e}$ alors $A$ est homogène.

Démonstration. Si $F_{e}=\operatorname{Aut}_{K}(A)$ l'affirmation se trivialise (Proposition 5.10). Si $F_{e} \neq \operatorname{Aut}_{K}(A)$ alors $U \neq\{0\}$ et il existe $\phi \in \operatorname{Aut}_{K}(A)$ tel que $\phi(e) \neq e$. Posons $\phi(e)=e_{\sigma}$ et soient $\theta \in U, f \in F_{e}$ tel que $f(\sigma)=\theta$. On a $(f \circ \phi)(e)=e_{\theta}$.

Remarque 5.14. $A$ est homogène si et seulement si $\operatorname{Aut}_{K}(A) / F_{e} \rightarrow \operatorname{Ip}(A)$ est bijective. Dans ce cas, $F_{e}$ est normal si et seulement si pour tout $f \in F_{e}$ la restriction à $U$ est égale à l'identité (Proposition 5.11).

Proposition 5.15. Soit $A$ homogène. Si $F_{e} \neq \mathrm{Aut}_{K}(A)$ les conditions suivantes sont équivalentes:

(a) $F_{e}$ est normal;

(b) pour tout $f \in F_{e}, u \in U$ et $v \in V$ on a $f(u)=u$ et $f(v)=v+v^{\prime}$ avec $v^{\prime} \in \operatorname{Ann}_{A}(A)$.

Démonstration. L'implication $b \Rightarrow a$ résulte de la Remarque 5.14. Pour ce qui est de la réciproque, de $u v=f(u v)=u f(v)$ on déduit que $f(v)=v+v^{\prime}$ avec $u v^{\prime}=0$ c'est-à-dire que $v^{\prime} U=\{0\}$. Il suffit donc d'appliquer la Proposition 3.6.

En général dans les algébres à idempotent et non associatives on ne sait pas caractériser celles qui sont homogènes. Pour les algèbres BJF le problème reste ouvert.

Nous avons cependant les résultats suivants:

Théorème 5.16. Les conditions suivantes sont équivalentes:

(a) A est quasi-orthogonale;

(b) $P_{e}$ est un sous-ensemble de $\operatorname{Aut}_{K}(A)$;

(c) A est homogène et tout $\phi \in \operatorname{Aut}_{K}(A)$ se décompose de façon unique: $\phi=\phi_{\sigma} \circ f$ avec $f \in F_{e}$.

Démonstration. L'équivalence $a \Leftrightarrow b$ découle de la définition de quasi-orthogonalité et de la Proposition 5.2. Quant à l'implication $b \Rightarrow c$, elle résulte $\operatorname{de}_{e} \subset \operatorname{Aut}_{K}(A)$; puis, si $\phi \in \operatorname{Aut}_{K}(A)$, il suffit de poser $f=\phi_{\sigma}^{-1} \circ \phi$. La décomposition est unique car si $\phi_{\sigma} \circ f=$ $\phi_{\theta} \circ g$, en appliquant l'identité sur e on obtient $\sigma=\theta$ et a fortiori $f=g$. Enfin, montrons que $c \Rightarrow a$ : soit $\sigma \in U$; comme $A$ est homogène il existe $\phi \in$ Aut $_{K}(A)$ tel que $\phi(e)=e_{\sigma}$. Soit 
$f \in F_{e}$ tel que $\phi=\phi_{\sigma} \circ f$; on a $\phi_{\sigma}=\phi \circ f^{-1}$ donc $\phi_{\sigma}$ est un automorphisme d'algèbres d'où $U^{2}(U V)=\{0\}$ (Proposition 5.2). Ceci prouve que $A$ est quasi-orthogonale.

Proposition 5.17. Les conditions suivantes sont équivalentes:

(a) A est orthogonale;

(b) $P_{e}$ est stable par Pinvolution $\operatorname{Aut}_{K}(A) \rightarrow \operatorname{Aut}_{K}(A), \phi \rightarrow \phi^{-1}$.

Démonstration. Ceci résulte de la Proposition 5.5 et du Théorème 5.16.

Théorème 5.18. Les conditions suivantes sont équivalentes:

(a) A est fortement-orthogonale;

(b) $P_{e}$ est un sous-groupe de $\operatorname{Aut}_{K}(A)$;

Dans ces conditions, l'application $\operatorname{Aut}_{K}(A) / F_{e} \rightarrow P_{e},[\phi] \rightarrow \phi_{\sigma}, \phi(e)=e_{\sigma}$ est bijective, $P_{e}$ est un sous-groupe normal et les groupes $\operatorname{Aut}_{K}(A) / P_{e}$ et $F_{e}$ sont isomorphes.

Démonstration. Pour l'équivalence, il suffit d'appliquer le Corollaire 5.8. Pour ce qui est du reste, comme $A$ est homogène (Théorème 5.16), l'application $\operatorname{Aut}_{K}(A) / F_{e} \rightarrow P_{e}$, $[\phi] \rightarrow \phi_{\sigma}, \phi(e)=e_{\sigma}$ est bijective car elle est la composée des bijections $\operatorname{Aut}_{K}(A) / F_{e} \rightarrow$ $\operatorname{Ip}(A),[\phi] \rightarrow \phi(e)$ et $\operatorname{Ip}(A) \rightarrow P_{e}, e_{\sigma} \rightarrow \phi_{\sigma}$ (5.14). Enfin, soient $\phi \in \operatorname{Aut}_{K}(A)$ et $\phi_{\theta} \in P_{e}$; il existent $f \in F_{e}$ et $\phi_{\sigma} \in P_{e}$ tels que $\phi=\phi_{\sigma} \circ f$ (Théorème 5.16). On a $\phi \circ \phi_{\theta}=\phi_{\sigma} \circ f \circ \phi_{\theta}=$ $\phi_{\sigma} \circ \phi_{f(\theta)} \circ f$ (Proposition 5.10); mais $\phi_{\sigma} \circ \phi_{f(\theta)}=\phi_{f(\theta)} \circ \phi_{\sigma}$ (Corollaire 5.8) d'où $\phi \circ \phi_{\theta}=$ $\phi_{f(\theta)} \circ \phi$; ceci prouve que $P_{e}$ est normal et de ce fait que $\operatorname{Aut}_{K}(A)=P_{e} F_{e}$ (Théorème 5.16) d'où l'somorphisme entre les groupes $\operatorname{Aut}_{K}(A) / P_{e}$ et $F_{e}$.

\section{Remarques sur les dérivations}

Nous ne pouvons qu'exprimer notre découragement face au caractère presque impénétrable de la structure des dérivations d'une algèbre BJF.

Néanmoins, nous présentons ici quelques résultats.

Si $d: A \rightarrow A$ est une dérivation, de l'identité $e^{2}=e$ on déduit que $d(e) \in U$; si de plus, $d(e)=0$ alors $d(U) \subset U$ et $d(V) \subset V$.

Proposition 6.1. Soit $\sigma \in U$. Les conditions suivantes sont équivalentes:

(a) $\sigma^{2}=0$;

(b) $\phi_{\sigma}-l$ est une dérivation;

(c) $\sigma \in \operatorname{Inv}\left(\phi_{\sigma}\right)$.

Et sous ces conditions $\phi_{\sigma}$ est un automorphisme.

Démonstration. Que $a \Leftrightarrow c$ est immédiat. Or, $b \Rightarrow a$ car $\left(\phi_{\sigma}-l\right)(e) \in U$. Montrons que $a \Rightarrow b$; toutes les identités qui suivent sont justifées par les résultats de 3.3 et de 3.5. Si $\sigma^{2}=0 \quad$ alors $\quad\left(\phi_{\sigma}-l\right)(e)=\sigma, \quad\left(\phi_{\sigma}-l\right)(u)=2 \sigma u \quad$ et $\quad\left(\phi_{\sigma}-l\right)(v)=-2 \sigma v$. On a 
$\left(\phi_{\sigma}-l\right)\left(u^{2}\right)=-2 \sigma u^{2}=4 u(\sigma u)=2 u(2 \sigma u)=2 u\left(\phi_{\sigma}-l\right)(u) ; \quad$ de même, $\quad\left(\phi_{\sigma}-l\right)\left(v^{2}\right)=$ $\left(\phi_{\sigma}-l\right)\left(\alpha_{v} e\right)=\alpha_{v}\left(\phi_{\sigma}-l\right)(e)=\alpha_{v} \sigma=-4 v(\sigma v)=2 v\left(\phi_{\sigma}-l\right)(v) ; \quad$ enfin, $\quad\left(\phi_{\sigma}-l\right)(u v)=2 \sigma(u v)=$ $u(-2 \sigma v)+v(2 \sigma u)=u\left(\phi_{\sigma}-l\right)(v)+v\left(\phi_{\sigma}-l\right)$. Donc, $\phi_{\sigma}-l$ est une dérivation. La dernière affirmation est immédiate.

Proposition 6.2. Soit $d$ une dérivation et $\phi=l+d$. Si $\phi \in P_{e}$ alors $\phi$ est un automorphisme. La réciproque n'est pas vraie.

Démonstration. Posons $d(e)=\sigma$. On a $\phi(e)=e+\sigma$ d'où $\phi=\phi_{\sigma}$ avec $\sigma^{2}=0$. Il suffit donc d'appliquer 6.1. Pour ce qui est de la deuxième affirmation, il est clair que $l+d$ est un automorphisme si et seulement si $d(x)^{2}=0$ pour tout $x$. Soient $A$ l'algèbre BJF de base $\left\{e, u, v^{\prime}, v\right\}$ et de table $e^{2}=e, e u=\frac{1}{2} u, u^{2}=v^{\prime}$, les autres produits étant nuls et $d: A \rightarrow A$ l'application linéaire définie par $d(e)=d(u)=d\left(v^{\prime}\right)=0, d(v)=v$. Cette application est une dérivation et $l+d$ est un automorphisme qui n'est pas une transformation de Peirce.

Nous tenons à remercier vivement le Referee de sa lecture attentive de notre article ainsi que de ses remarques très pertinentes.

\section{BIBLIOGRAPHIE}

1. S. Bernstejn, Démonstration mathématique de la loi d'hérédité de Mendel, $C$. $R$. $A c$. Sc. Paris 177 (1923), 528-531.

2. S. Bernstein, Principle de stationarité et généralisation de la loi de Mendel, $C . R . A c$. Sc. Paris 177 (1923), 581-584.

3. S. Bernstein, Solution of a mathematical problem connected with the theory of heredity, Ann. Sc. de l'Ukranie 1 (1924), 83-114 (en russe). Traduction anglaise: Ann. of Math. Statistics 13 (1942), 53-61.

4. M. T. Alcalde, C. Burgueño, A. Labra et A. Micali, Sur les algèbres de Bernstein, Proc. London Math. Soc. (3) 58 (1989), 51-68.

5. I. M. H. Etherington, Genetic Algebras, Proc. Roy. Soc. Edinburgh 59 (1939), 242-258.

6. P. Holgate, Genetic algebras satisfying Bernstein stationarity principle, J. London Math. Soc. 9 (1975), 613-624.

7. C. Mallol et A. Micali, Sur les algèbres de Bernstein II, Linear algebra and its applications 117: 11-19 (1989).

8. C. Mallol, A. Micali et M. Ouattara, Sur les algèbres de Bernstein IV, Linear Algebra Appl. 158 (1991), 1-26.

9. A. Micall et M. Ouattara, Sur les algèbres de Jordan Génétiques, Algebras, Groups and Geometries (1990), à paraître. 
10. A. Worz-Busekros, Algebras in genetics (Lecture Notes in Biomathematics 36, SpringerVerlag, Berlin, 1980), 21.

Departamento de Matematicas

Facultad de Ciencias

Universidad de Chile

CASILLA 653

SANTIAGo 1

Chile

Dept. de Mathématiques

Université de MontPellier II

Place E Bataillon

34095 MontPEllier 05

FRANCE
Mathématiques Appliquées

ET INFORMATIQue

Université de MontPellier III

B.P. 5043

34032 MontPelLier 1

FrancE

Dept. de Mathématiques

Universite De Montpellier II

Place E Batalllon

34095 Montpellier 05

FRANCE 